\title{
O USO DA INFORMÁTICA NA REDE BÁSICA E HOSPITALAR DA CIDADE DE RIBEIRÃO PRETO (S.P.)
}

\author{
Cristina Maria Galvão * \\ Namie Okino Sawada *
}

GALVÃO, C. M.; SAWADA, N. O. O uso da informática na rede básica e hospitalar da cidade de Ribeirão Preto (S.P.). Rev.latino-am.enfermagem, Ribeirão Preto, v. 4, $\mathrm{n}^{\circ}$ especial, p. 51-60, abril 1996.

O presente trabalho tem como objetivo relatar o uso da informática na rede básica e hospitalar na cidade de Ribeirão Preto (SP), A coleta de dados foi realizada em sete hospitais gerais e na Secretaria Municipal de Saúde, por meio de entrevistas estruturadas com os responsáveis pela informática nessas instituições, Efetuou-se uma análise descritiva e matemático-estatística, utilizando freqüência absoluta e porcentagem. Os dados evidenciaram a utilização de computadores em atividades da área administrativa das instituições pesquisadas, entretanto, existem projetos de implantação desta tecnologia na área assistencial a médio e longo prazo.

UNITERMOS: informática, rede básica e hospitalar

A sociedade atual sofre grande influência da tecnologia de informação. A informática está presente no nosso cotidiano através dos microcomputadores, dos caixas eletrônicos dos bancos, videogames, fax etc.

Segundo Sabbatini apud GUIMARÃES ${ }^{4}$ a utilização da informática na área da saúde, no Brasil, apresenta um atraso considerável quando comparado a outros países, entretanto, a partir da década de 80 observa-se um avanço importante nessa área.

\footnotetext{
* Enfermeira, Assistente junto ao Departamento de Enfermagem Geral e Especializada da Escola de Enfermagem de Ribeirão Preto da Universidade de São Paulo
} 
BRASIL $^{2}$ define a informática em saúde como área do saber, que trata das aplicações e usos das ferramentas de automação de processamento de dados e informações, nos vários segmentos de atividades relacionadas à saúde do indivíduo e da coletividade.

De acordo com GUIMARÃES ${ }^{4}$ a utilização do computador na saúde iniciou na área hospitalar administrativa para registro da admissão do cliente, busca de leitos, internação e estudos estatísticos. Ressalta ainda que este recurso pode estender-se igualmente para a área assistencial.

O uso da informática na área da saúde proporciona uma melhoria na qualidade da assistência prestada, bem como a racionalização dos serviços e recursos humanos ${ }^{3,4,7}$.

Para ÉVORA ${ }^{3}$, na enfermagem, a informática pode ser utilizada em quatro áreas: administração; assistência; ensino e pesquisa. Segundo a autora, o uso do computador na área da administração proporcionaria a construção de instrumentos informacionais, os quais auxiliariam o enfermeiro no gerenciamento de uma unidade, assegurando um planejamento adequado dos recursos humanos, materiais e financeiros. Na assistência, o computador facilitaria a coleta, armazenamento e o processamento de informações que auxiliam no tratamento do paciente. Além disso, ocorre a liberação do enfermeiro das atividades burocráticas, permitindo uma maior disponibilidade para o planejamento da assistência, observando o paciente como um todo. No ensino, viabilizaria instruções individualizadas, facilitaria a transferência da teoria à prática, bem como, a capacidade do aluno em solucionar os problemas. $\mathrm{O}$ computador poderá auxiliar todo o processo de pesquisa, desde a coleta de dados até as análises, assim como a recuperação de informações, edição de textos, bancos de dados, entre outros.

Motivadas por essas considerações e com o propósito de conhecer a utilização da tecnologia computacional na área da saúde na cidade de Ribeirão Preto, o presente trabalho tem como objetivo relatar o uso da informática na rede básica e hospitalar dessa cidade.

\section{METODOLOGIA}

\subsection{Local de coleta de dados}

O presente trabalho foi desenvolvido em sete hospitais gerais de Ribeirão Preto sendo um governamental e seis particulares; desses, três atendem particular, convênios e Sistema Único de Saúde (SUS) e três somente convênios e particular, e na Secretaria Municipal de Saúde (SMS). Foi excluído um hospital geral por não possuir um Centro de Processamento de Dados. 


\subsection{Amostra}

Fizeram parte deste estudo dois diretores administrativos, cinco encarregados pelo Centro de Processamento de Dados (CPD) de instituições hospitalares e um coordenador do sistema integrado de informações e informatização da SMS.

\subsection{Coleta de dados}

A coleta de dados foi realizada através de entrevista estruturada com questões abertas (anexo A). As entrevistas foram previamente agendadas, com duração de aproximadamente 30 minutos.

\subsection{Análise dos dados}

Com os dados coletados foi realizada uma análise descritiva e matemáticoestatística, utilizando freqüência absoluta e porcentagem.

\section{RESULTADOS E DISCUSSÃO}

A Tabela 1 apresenta a distribuição das instituições de saúde pesquisadas segundo número de leitos, número de internações e atendimentos médicos mensais de acordo com os entrevistados.

Em relação aos recursos humanos contratados especificamente para a área da informática encontrou-se um número reduzido de profissionais especializados, sendo que a maioria desses são programadores e operadores. Acredita-se que este fato ocorre, devido a terceirização dos serviços de informática encontrada em seis das instituições estudadas. Em duas destas, a terceirização ocorre através de vínculo contínuo com empresas de capital estatal e particular enquanto, que as outras são empresas particulares que realizam assessorias temporárias.

$\mathrm{Na}$ Tabela 2 pode-se visualizar a distribuição dos setores informatizados nas instituições de saúde estudadas conforme relato dos entrevistados. Constatou-se que a maioria destes setores pertence à área administrativa, sendo que os setores almoxarifado, recursos humanos, financeiro e farmácia, tiveram o maior índice de porcentagem (17,95\%),

Em relação às finalidades sobre o uso da informática nas instituições instigadas, na Tabela 3, pode-se observar o conjunto levantado, sendo que rapidez na obtenção de informações alcançou o maior índice de porcentagem (34,78\%), Esses achados vem ao encontro dos benefícios apontados por ÉVORA ${ }^{3}$ no que tange a rapidez e facilidade na documentação das atividades através de sistemas informatizados. 
TABELA 1 - DISTRIBUIÇÃO DAS INSTITUIÇÕES DE SAÚDE PESQUISADAS, SEGUNDO NÚMERO DE LEITOS, NÚMERO DE INTERNAÇÕES E ATENDIMENTOS MÉDICOS MENSAIS DE ACORDO COM OS ENTREVISTADOS

\section{CARACTERÍSTICAS}

\begin{tabular}{c|c|c|c}
\hline $\begin{array}{c}\text { INSTITUIÇÃO DE } \\
\text { SAÚDE }\end{array}$ & $\begin{array}{c}\text { NÚMEROS DE } \\
\text { LEITOS }\end{array}$ & $\begin{array}{c}\text { NÚMERO DE } \\
\text { INTERNAÇÕESI } \\
\text { MÊS }\end{array}$ & $\begin{array}{c}\text { NÚMERO DE } \\
\text { ATENDIMENTOI } \\
\text { MÊS }\end{array}$ \\
\hline SMS & --- & --- & 7.100 \\
A & 657 & 2.800 & 42.700 \\
B & 110 & 300 & 2.200 \\
C & 287 & 1.300 & 30.000 \\
D & 200 & 800 & 4.800 \\
E & 65 & 350 & 1.600 \\
F & 200 & 519 & 1.059 \\
G & 98 & 450 & 1.100 \\
\hline
\end{tabular}

SMS - Secretaria Municipal da Saúde (compreende aproximadamente 28 unidades de saúde)

A - Hospital Governamental (compreende 2 unidades)

B,C,D - Hospital Particular (com atendimento particular, convênios e SUS)

E,F,G - Hospital Particular (com atendimento particular e convênios)

TABELA 2 - DISTRIBUIÇÃO DOS SETORES INFORMATIZADOS DAS INSTITUIÇÕES DE SAÚDE PESQUISADAS, SEGUNDO OS ENTREVISTADOS

\begin{tabular}{lrrr}
\hline SETORES & F & $\%$ \\
INFORMATIZADOS & 7 & 17,95 \\
\hline Almoxarifado & 7 & 17,95 \\
Farmácia & 7 & 17,95 \\
Recursos Humanos & 6 & 15,40 \\
Recepção (registro do paciente, abertura de prontuário médico) & 7 & 17,95 \\
Financeiro (contabilidade, faturamento, patrimônio e orçamento) & 1 & 2,56 \\
Controle de leitos & 1 & 2,56 \\
Agendamento de consultas & 2 & 5,12 \\
Controle de infecção hospitalar & 1 & 2,56 \\
Exames com alta tecnologia (radiologia, tomografia) & $\mathbf{3 9}$ & $\mathbf{1 0 0 , 0 0}$ \\
\hline TOTAL & &
\end{tabular}




\section{TABELA 3 - DISTRIBUIÇÃO DAS FINALIDADES SOBRE $O$ USO DA INFORMÁTICA NAS INSTITUIÇÕES DE SAÚDE PESQUISADAS, SEGUNDO OS ENTREVISTADOS}

\begin{tabular}{lcc}
\hline FINALIDADES & F & $\%$ \\
\hline Rapidez na obtenção de informações & 8 & 34,78 \\
Segurança e precisão das informações & 3 & 13,04 \\
Banco de dados para pesquisa & 2 & 8,70 \\
Controle financeiro & 3 & 13,04 \\
Controle de serviços & 2 & 8,70 \\
Controle e racionalização de recursos humanos & 3 & 13,04 \\
Melhoria do atendimento ao cliente & 2 & 8,70 \\
\hline TOTAL & $\mathbf{2 3}$ & $\mathbf{1 0 0 , 0 0}$ \\
\hline
\end{tabular}

Pode-se observar, na Tabela 4, as vantagens e desvantagens sobre o uso da informática nas instituições de saúde estudadas, apontadas pelos entrevistados. Em relação às vantagens, novamente rapidez na obtenção de informações alcançou o maior índice de porcentagem (34,79\%), ficando em segundo lugar a racionalização dos serviços (13,05\%).

Dentro das vantagens apontadas, salienta-se a questão da redução dos custos. Conforme artigo de MORAES ${ }^{6}$ "um estudo recente mostrou que o gasto anual na área da saúde nos Estados Unidos poderia ser reduzido em mais de US $\$ 36$ bilhões se algumas tecnologias de informação fossem adotadas em rede nacional". Acrescenta o artigo que "aparentemente, o sistema de saúde é o único serviço do país que ainda conserva seus arquivos em papel. Por esta razão, médicos e enfermeiras passam mais de $50 \%$ do seu tempo envolvidos em atividades burocráticas e apenas 35\% atendendo pacientes". A racionalização de serviços e recursos humanos também foram vantagens levantadas neste estudo, conforme visualiza-se na Tabela 4. 
TABELA 4 - DISTRIBUIÇÃO DAS VANTAGENS E DESVANTAGENS SOBRE O USO DA INFORMÁTICA NAS INSTITUIÇÕES DE SAÚDE PESQUISADAS, SEGUNDO OS ENTREVISTADOS

\begin{tabular}{|c|c|c|c|c|c|}
\hline VANTAGENS & $\mathbf{F}$ & $\%$ & DESVANTAGENS & $\mathbf{F}$ & $\%$ \\
\hline Facilita a tomada de decisões & 1 & 4,35 & $\begin{array}{l}\text { Rapidez na obsolescência } \\
\text { dos equipamentos }\end{array}$ & 1 & 9,08 \\
\hline $\begin{array}{l}\text { Permite gerenciamento } \\
\text { global }\end{array}$ & 2 & 8,69 & $\begin{array}{l}\text { Usuários apresentam } \\
\text { dificuldades na compreensão } \\
\text { do processamento das } \\
\text { operações }\end{array}$ & 2 & 18,19 \\
\hline Reduz custos & 2 & 8,69 & $\begin{array}{l}\text { Usuários } \\
\text { dificuldades em upresentam } \\
\text { computador }\end{array}$ & 2 & 18,19 \\
\hline $\begin{array}{l}\text { Rapidez na obtenção de } \\
\text { informações }\end{array}$ & 8 & 34,79 & $\begin{array}{l}\text { Escassez de profissionais } \\
\text { qualificados }\end{array}$ & 1 & 9,08 \\
\hline Racionalização de serviços & 3 & 13,05 & Alto custo & 2 & 18,19 \\
\hline $\begin{array}{l}\text { Racionalização de recursos } \\
\text { humanos }\end{array}$ & 1 & 4,35 & Exige constante atualização & 1 & 9,08 \\
\hline $\begin{array}{l}\text { Segurança e precisão das } \\
\text { informações }\end{array}$ & 2 & 8,69 & $\begin{array}{l}\text { Ausência de sistema de } \\
\text { apoio, quando ocorre } \\
\text { interrupções nos sistemas } \\
\text { informatizados }\end{array}$ & 2 & 18,19 \\
\hline $\begin{array}{l}\text { Aumenta a qualidade dos } \\
\text { serviços }\end{array}$ & 2 & 8,69 & & & \\
\hline $\begin{array}{l}\text { Permite o levantamento de } \\
\text { dados para pesquisa }\end{array}$ & 1 & 4,35 & & & \\
\hline $\begin{array}{l}\text { Auxilia a avaliação dos } \\
\text { serviços }\end{array}$ & 1 & 4,35 & & & \\
\hline TOTAL & 23 & 100,00 & & 11 & 100,00 \\
\hline
\end{tabular}

As desvantagens constatadas nas instituições de saúde estudadas com os maiores índices de porcentagem (18,19\%) foram: dificuldades apresentadas pelos usuários na compreensão do processamento das operações bem como na utilização do computador, ausência de sistemas de apoio quando ocorrem interrupções nos sistemas informatizados e o alto custo.

ADAMS $^{1}$ ressalta a importância dos profissionais da área de saúde, especificamente os enfermeiros, obterem conhecimento teórico-prático sobre informática possibilitando uma melhor compreensão desta tecnologia, bem como uma melhor utilização do computador seja para otimizar a assistência prestada ao cliente, como também a racionalização do processo de trabalho.

Quanto às perspectivas relatadas sobre o uso da informática nas instituições estudadas, verificou-se que todas possuem um projeto de implementação de sistemas informatizados voltados para a assistência direta ao cliente (por exemplo: sistemas para exames laboratoriais; controle de cirurgias, antimicrobianos, prontuários, dentre outros), entretanto, esse avanço enfrenta vários problemas como: financeiro, treinamento de recursos humanos e resistência de profissionais da área da saúde na utilização dessa tecnologia. 
Das instituições estudadas constatou-se que esses projetos apresentam fases distintas, sendo que alguns estão em início de implementação e outros apenas em estudo.

Salienta-se que apenas a Secretaria Municipal de Saúde possui um sistema integrado de atendimento ao usuário que proporcionar um cadastramento único, agendamento informatizado, histórico clínico computadorizado e estatísticas ambulatoriais, o qual será Implementado no corrente ano, nas unidades de saúde.

Os dados do presente estudo evidenciaram que apesar de ser a área administrativa das instituições estudadas, a que mais sofreu um processo de informatização, visualizou-se um avanço na área assistencial. Concorda-se com o pensamento de LUIS et $\mathrm{al}^{5}$ "será necessário encontrar um ponto de equilíbrio entre as formas de organização de trabalho e o uso do instrumental automatizado, investindose nas relações humanas chefes-liderados e na busca de alternativas para rehumanização dos processos tecnológicos incorporados ao trabalho", por entender que a tecnologia pode interferir nessas relações.

\section{CONSIDERAÇÕES FINAIS}

O processo de informatização na área da saúde poderá trazer inúmeros benefícios para a melhoria da qualidade da assistência prestada ao cliente, seja em atividades administrativas como em atividades assistenciais.

O processo de informatização das atividades assistenciais das instituições estudadas deverá ocorrer a médio e longo prazo, Assim, acredita-se que os profissionais da área da saúde necessitam refletir sobre a inserção desta tecnologia na sua prática diária.

Ressalta-se ainda, a importância do preparo formal para a utilização da informática, com o propósito de capacitar os profissionais na compreensão, manuseio e emprego desta tecnologia de informação.

\section{THE USE OF COMPUTER TECHNOLOGY IN BASIC AND HOSPITAL NETWORKS IN THE CITY OF RIBEIRÃO PRETO (BRAZIL)}

The purpose of this study is to report the use of computer technology in basic and hospital networks in the city of Ribeirão Preto (SP), Data were collected in seven general 
hospitals and in the Municipal Bureau of Health. Descriptive and mathematicalstatistical analyzes were effected using absolute frequency and percentage. The results showed the utilization of the computer technology in the activities of some institutions administrative area; however, there are long and short term projects to introduce this technology in nursing care.

UNITERMS: computer technology, basic and hospital networks

\section{EL USO DE LA INFORMÁTICA EN LA RED BÁSICA Y HOSPITALARIA DE LA CIUDAD DE RIBEIRÃO PRETO (S.P.)}

El presente estudio tiene como objetivo relatar el uso de la informática en la Red Básica y Hospitalaria de la ciudad de Ribeirão Preto (S.P.). La coleta de datos fue realizada en siete hospitales generales en la Secretaría Municipal de Salud, a través de entrevistas estructuradas con los responsables por la informática en estas instituciones. Fue efectuado un análisis descriptivo y matemático-estadístico, utilizando frecuencia absoluta y porcentaje. Los datos evidenciaran la utilización de computadores en actividades del área administrativa de las instituciones, entretanto, hay proyectos de implantación de esta tecnología en el área asistencial a medio y largo plazo.

UNITERMOS: informática, red básica y hospitalaria

\section{REFERÊNCIAS BIBLIOGRÁFICAS}

01. ADAMS, G. A. Computer technology: its impact on nursing. Nurs.Adm.Q., v. 10, n. 2, p. 21-33,1986.

02. BRASIL. Ministério da Ciência e Tecnologia. Conselho Nacional de Informática e Automação. Secretaria Especial de Informática. Proposta de plano setorial de informática em saúde: relatório da comissão especial. Brasília, 1988.

03. ÉVORA, Y. D. M. Enfermagem e informática. Tendências atuais e perspectivas futuras. Ribeirão Preto, 1993. p. 230. Tese (Doutorado) - Escola de Enfermagem de Ribeirão Preto, Universidade de São Paulo.

04. GUIMARÃES, S. M. A informática na enfermagem: introduzindo o computador na estratégia de ensino e assistência de enfermagem. Porto Alegre, 1988. p. 243. Dissertação (Mestrado) - Pontifícia Universidade Católica do Rio Grande do Sul. 
05. LUIS M. V.; SCOCHI, C. G. S. ; ATZINGEN, R.H.V. Reflexões sobre a inserção da informática na profissão de enfermagem. Rev.Gaúch.Enfermagem, v. 13, n. 1, p. 37-40, jan. 1992.

06. MORAES, M. Uso de computador em hospitais pode economizar US\$ 36 bilhões ao ano E.U.A. Folha de São Paulo. São Paulo, 02 de março. 1994. p.6-2.

07. SILVA, C.M. Informatização de tarefas administrativo-burocráticas de enfermagem relacionadas com o preparo para cirurgias eletivas: uma proposta. São Paulo, 1991. p. 87. Dissertação (Mestrado) - Escola de Enfermagem, Universidade de São Paulo. 
ANEXO A

Nome da Instituição de Saúde:

Tipo:

$\mathrm{N}^{\circ}$ de Leitos: $\quad \mathrm{N}^{\circ}$ de Atendimentos:

Recursos Humanos contratados para utilização da informática:

1) Esta instituição realiza a informatização de informações?

2) No caso afirmativo, quais são os setores informatizados?

3) Qual a finalidade da informatização nestes setores?

4) Na sua opinião, quais são as vantagens e desvantagens da informatização.

5) Quais são as perspectivas que você visualiza nesta área? 